\title{
A Review on New Technique for Embedding Image into Audio as a Watermark using DCT
}

\author{
Yatish Y. Jani \\ Marwadi Education \\ Foundation's Faculty of \\ P.G.Studies \& Research in \\ Engineering \& Technology, \\ Rajkot, Gujarat, India
}

\author{
Yagnesh J. Parmar \\ C.U.Shah collage of \\ engineering \& technology, \\ Wadhwan, Gujarat,India
}

\author{
Chintan J. Kavathiya \\ Marwadi Education \\ Foundation's Faculty of \\ P.G.Studies \& Research in \\ Engineering \& Technology, \\ Rajkot, Gujarat, India
}

\begin{abstract}
Globalization and internet are the two main reasons for rapidly spreading of the multimedia information and due to that ownership and copyright of multimedia files are not usually protected by the providers. Digital watermarking is one of the best ways for copyright protection. Researchers are trying to invent new techniques that increase the security, Robustness and many more things. In this paper we proposed a new technique based on DCT. In this technique bits used as a watermark are inserted in whole audio file. Watermarked bits are inserted in all frames' first bit in such a way that it should not affect the original media file. This method is comparatively easy to implement, more robust and can carry more data as a watermark.
\end{abstract}

\section{Keywords}

Audio watermarking, DCT, DWT, Echo Hiding

\section{INTRODUCTION}

Increase the use of internet caused major changes in global society. Digital watermarking has to embed some of information into a digital media for protecting it against copyright and ownership infringements and other unauthorized applications. A lot of works has been done on digital watermarking of various media such as image and video, but this particular review will focus on digital watermarking of audio file. Digital Audio files are particularly the most abused for copyright infringements because they can be downloaded, shared or copied easily.

Audio watermarks are special signals (text, symbols, image, etc.) embedded into original audio file. These signals are extracted by detection mechanisms. Audio watermarking schemes depend on the human auditory system. However, human ear is much more sensitive than other sensory motors. Thus, good audio watermarking schemes are difficult to design. A lot of recent works have proposed solutions to this problem of copyright infringement. In recent years, many watermarking methods have been proposed for audio signals. These audio watermarking methods use different techniques such as spread spectrum [11]-[13], echo hiding [5], [6], [14][18], Frequency Domain techniques, etc.

In this paper, we propose a new DCT based watermarking scheme for audio signals. In the proposed scheme, the host audio segment is divided in to number of frames. The number of frames should be equal to the size of the watermark and then the DCT coefficients of the sub segments are calculated. After that we also calculate the DCT for the watermark. Then insert the bits of watermark in the frames one by one sequentially. Then do the inverse DCT. At the extraction side we do the same framing then subtract the embedded audio signal from the original audio signal so we can get the extracted watermark. The new scheme has higher robustness against conventional attacks. The remainder of the paper is organized as follows.

Features of audio watermarking are explained in section II. Different techniques are listed with their advantages and disadvantages are explained in section III. The new embedding and decoding scheme is presented in Section IV.

\section{FEATURES OF AUDIO \\ WATERMARKING}

There are several criteria that need to be satisfied for effective application of watermarking technology. These are robustness, imperceptibility, security and computational complexity. They are discussed below.

\subsection{Robustness}

Digital signal may undergo common signal processing operations such as filtering, sample re-quantization, noise, etc.

\section{Imperceptibility}

This refers to imperceptibility of embedded watermark within the host signal. The signal-to-noise ratio of the watermark versus the host signal is the measure of the perceptual quality. The embedded watermark should not affect much to the host signal.

\subsection{Watermark security}

Watermark security refers to the inability by unauthorized users to have access to the watermarked audio signal.

\subsection{Computational complexity}

This refers to the processing required to embed data into the host signal and or to extract data from the signal.

\section{AUDIO WATERMARKING TECHNIQUES}

Audio watermarking techniques can be classified in two types based on domain. They are spatial domain and frequency domain. Detailed description with advantages and disadvantages about these two techniques are given below.

\subsection{Spatial Domain Technique}

Techniques in spatial domain have generally following characteristics.

- The watermark is directly applied to the host signal. 
- No transforms are applied on the host signal during watermark embedding.

- Embedding is based on simple operations in the pixel domain.

The main strengths of spatial domain methods are that they are conceptually simple and have very low computational complexity. However, they also exhibit a major drawback that robustness of spatial domain technique is low as compare to frequency domain technique.

There are two techniques mostly popular in spatial domain. They are:

- $\quad$ Least significant bit method (LSB)

- $\quad$ Echo hiding method

Deast Significant Bit (LSB) Technique: The most straightforward method of watermark embedding would be to embed the watermark into the least significant bits of the cover object. In this method, a smaller object may be embedded multiple times. Even if most of these are lost due to attacks, a single surviving watermark would be considered a success. Although it may survive transformations such as cropping, any addition of noise or lossy compression is likely to defeat the watermark. LSB has some drawbacks. It would effect on the original signal. Also, once the algorithm is discovered, the embedded watermark could be easily modified.

$>$ Echo hiding Technique: This is another popular method of spatial domain technique. In this method the watermark signal is hided in the echo of the host signal. Due to this it is not easily detected. But in this method also there is no transform technique is used. So this technique is also not as highly robust against various attacks as frequency domain techniques.

\subsection{Frequency Domain Technique}

Frequency domain techniques can further divided in three parts.

- $\quad$ Fast Fourier Transform technique (FFT)

- Discrete Wavelet Transform technique (DWT)

- Discrete Cosine Transform technique (DCT)

$>$ Fast Fourier Transform technique: This technique is not mostly used for watermark embedding because in this technique it contains both even and

odd components of the signal. And for watermarking we need only even or we can say lower frequency components, Because if watermark added in lower frequency components then robustness of the host signal would be increased.

$>$ Discrete Wavelet Transform technique: Most of the researchers focus on embedding watermark in wavelet domain because watermarks in this domain are very robust. But there is one problem in this method is that it only embeds the watermark bits in the low energy bits. So there may be chances to detect the embed watermark from the Embedded signal.

$>$ Discrete Cosine Transform technique: The classic and still most popular domain for audio processing is that of the Discrete Cosine Transform, or DCT. In this technique watermark data bits are spread all over the host signal so that the robustness of the embedded signal is increased. In this method only low frequency or we can say even components are present so embedding using DCT gives the better result against the various attacks. For a 1-D discrete signal with the length of $N$, DCT is expressed as

$C(m)=a(m) \sum_{n=0}^{N-1} f(n)\left[\frac{\pi(2 n+1) m}{2 N}\right]$

Where $m=0 ; 1 ; 2 ; \cdots ; N-1$. For the 1-D signal, reverse DCT is represented as

$$
f(n)=\sum_{n=0}^{N-1} a(m) C(m) \cos \left[\frac{\pi(2 m+1) n}{2 N}\right]
$$

In (1) and (2), for $m=0 ; 1 ; 2 ; \cdots ; N-1, a(m)$

$$
a(m)= \begin{cases}\sqrt{\frac{1}{N}}, & \text { If } \mathrm{m}=0 \\ \sqrt{\frac{2}{N}}, & \text { If } \mathrm{m} \neq 0\end{cases}
$$

\section{PROPOSED TECHNIQUE}

Here is an effective audio watermarking technique which gives advance level of security and increasing the impeccability and robustness of the watermark. The planned method is divided into two parts, watermark embedding and watermark extraction process

\subsection{Watermark Embedding Process}

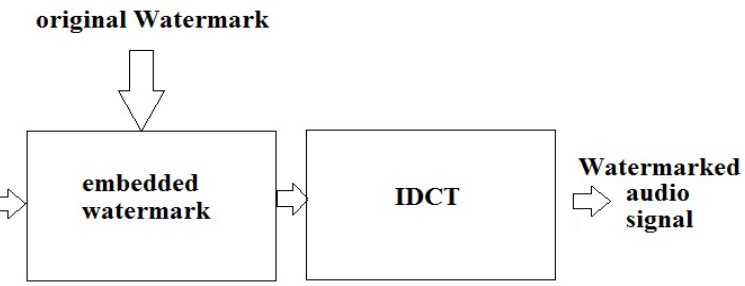

Fig. 1 DCT based watermark Embedding process

Step 1:

- In the embedding process, the original audio signal is divided into frames. The number of frame is equal to the size of the image.
- E.g. if the number of samples are 1000 and the image is $10 * 10$ then no. of frames must be equal to $1000 / 100=10$. 
Step 2:

- Select the image which you want to use as a watermark. Then resize the image in such form that our calculation for framing becomes easy.

Step 3:

- $\quad$ Take the Discrete Cosine Transform (DCT) of each frame and also the resized image. So the values of samples have ranges converted from -1 to 1 .

Step 4:

- Insert one by one all the pixel values of a watermark image in first sample of each frame sequentially. Here one new concept comes in picture. If we directly insert the pixel values in the host signal then it may create an audible noise.

- To overcome this problem we multiply the pixel value with one constant value named $\alpha$ and then we add it in to host signal. Choose the value of $\alpha$ such that the embedded signal looks similar as host signal and don't create any audible sound while playing it.

Step 5:

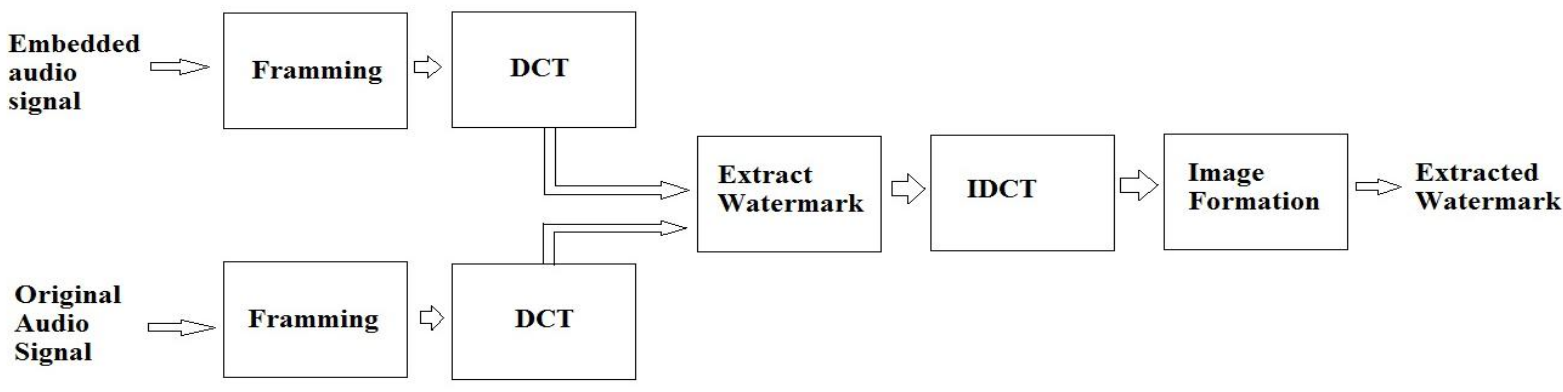

Fig. 2 DCT based watermark Extraction process

\section{CONCLUSION AND FUTURE WORK}

By this method as per the calculation the conclusion is that data used as a watermark can be more as compared to other methods without affecting the original audio file. Means more robust and more data can be used as a Watermark. In future this method can also be used for military purpose to send a secrete data and also it can be used for ownership protection.

\section{REFERENCES}

[1] Iynkaran natgunanathan, yong xiang, you rong, "robust patchwork based embedding and decoding scheme for digital audio watermarking", IEEE transaction on audio, speech and language processing, vol.20 October 2012.

[2] Hooman Nikmehr, Sina Tayefeh Hashemy, "A new approach to audio watermarking using discrete wavelet and cosine transforms" $1^{\text {st }}$ International Conference on communications Engineering, university of sistan \& baluchestan page no. 1-10, 22-24 December 2010.

[3] Ms.komal V.Goenka, Ms. Pallavi K. Patil, "overview of audio watermarking techniques ", international journal of emerging technology and advanced engineering ISSN 2250-2459, Volume 2, issue2, February 2012.

[4] Mayank Sharma, Savita Shiwani, "noise attack analysis on non-blind dwt watermarking algorithm" International Journal of Emerging Technology and Advanced
- Do Inverse DCT of each frame after inserting new values in first bit of each frame. After doing IDCT we get the final embedded signal.

\subsection{Watermark Extraction Process} Step 1:

- First step of extraction process is to divide the watermarked signal after different kind of attacks into frames as per the calculation shown in first step of embedding process.

Step 2:

- Do the DCT of each frame of the original audio signal and watermarked audio signal.

Step 3:

- Subtract the coefficient of the first bit of each frame of original audio signal from the embedded audio signal

Step 4:

- Extracted coefficients are arranged by doing the Inverse DCT. So as a result we get the Extracted Watermark image.
Engineering ISSN 2250-2459, ISO 9001:2008 Certified Journal, Volume 3, Issue 7, July2013.

[5] Acoust., vol. 69, no. 10, pp. 868-874, Oct. 2008.Y. Yorozu, M. Hirano, K. Oka, and Y. Tagawa, "Electron spectroscopy studies on magneto-optical media and plastic substrate interface," IEEE Transl. J. Magn. Japan, vol. 2, pp. 740-741, August 1987 [Digests 9th Annual Conf. Magnetics Japan, p. 301, 1982].

[6] O. T.-C. Chen and W.-C. Wu, "Highly robust, secure, and perceptual quality echo hiding scheme," IEEE Trans. Audio, Speech, Lang. Process., vol. 16, no. 3, pp. 629638, Mar. 2008

[7] Darshana Mistry, "Comparison of Digital Water Marking methods" International Journal on Computer Science and Engineering Vol. 02, No. 09, 2010, 2905-2909.

[8] Gaurav Chawla, Ravi Saini, Rajkumar Yadav, Kamaldeep, "Classification of Watermarking Based upon Various Parameters" International Journal of Computer Applications \& Information Technology Vol. I, Issue II, September 2012 (ISSN: 2278-7720).

[9] Yusuf Perwej, Firoj Parwej, Asif Perwej, “An Adaptive Watermarking Technique for the copyright of digital images and Digital Image Protection" The International Journal of Multimedia \& Its Applications (IJMA) Vol.4, No.2, April 2012 
[10] Ying Yang, Xingming Sun, Hengfu Yang "Removable visible image watermarking algorithm in the discrete cosine transform domain", Jul-Sep 2008.

[11] Z. Liu and A. Inoue, "Audio watermarking techniques using sinusoidal pattern based on pseudorandom sequence," IEEE Trans. Circuits Syst. Video Technol., vol. 13, no. 8, pp. 801-812, Aug. 2003.

[12] Valizadeh and Z. J. Wang, "Correlation-and-bit-aware spread spectrum embedding for data hiding," IEEE Trans. Inf. Forensics Security, vol. 6, no. 2, pp. 267-282, Jun. 2011.

[13] N. Cvejic and T. Seppänen, "Spread spectrum audio watermarking using frequency hopping and attack characterization," Signal Process., vol. 84, pp. 207-213, 2004 .

[14] B.-S. Ko, R. Nishimura, and Y. Suzuki, "Time-spread echo method for digital audio watermarking," IEEE Trans. Multimedia, vol. 7, no. 2, pp. 212-221, Apr. 2005.

[15] Y. Xiang, D. Peng, I. Natgunanathan, and W. Zhou, "Effective pseudonoise sequence and decoding function for imperceptibility and robustness enhancement in timespread echo based audio watermarking," IEEE Trans. Multimedia, vol. 13, no. 1, pp. 2-13, Feb. 2011.
[16] Y. Xiang, I. Natgunanathan, D. Peng, W. Zhou, and S. $\mathrm{Yu}$, "A dual-channel time-spread echo method for audio watermarking," IEEE Trans. Inf. Forensics Security, vol. 7, no. 2, pp. 383-392, Apr. 2012.

[17] Y. Erfani and S. Siahpoush, "Robust audio watermarking using improved TS echo hiding," Digital Signal Process., vol. 19, pp. 809-814, 2009.

[18] H. J. Kim and Y. H. Choi, "A novel echo-hiding scheme with backward and forward kernels," IEEE Trans. Circuit Syst. Video Technol., vol. 13, no. 8, pp. 885-889, Aug. 2003.

[19] X. Wang, W. Qi, and P. Niu, "A new adaptive digital audio watermarking based on support vector regression," IEEE Trans. Audio, Speech, Lang. Process., vol. 15, no. 8, pp. 2270-2277, Nov. 2007.

[20] I. K.Yeo and H. J. Kim, "Modified patchwork algorithm:A novel audio watermarking scheme," IEEE Trans. Speech Audio Process., vol. 11, no. 4, pp. 381386, Jul. 2003.

[21] Baras, N. Moreau, and P. Dymarski, "Controlling the inaudibility and maximizing the robustness in an audio annotation watermarking system," IEEE Trans. Audio, Speech, Lang. Process., vol. 14, no. 5, pp. 1772-1782, Sep. 2006. 